\title{
NEW GENERALIZATIONS OF JENSEN'S FUNCTIONAL EQUATION
}

\author{
HIROSHI HARUKI AND THEMISTOCLES M. RASSIAS
}

(Communicated by Palle E. T. Jorgensen)

ABstract. Let $f$ be an unknown entire function of a complex variable, and let $s, t$ be real variables. We consider Jensen's functional equation

$$
f\left(\frac{x+y}{2}\right)=\frac{f(x)+f(y)}{2},
$$

where $x, y$ are complex variables. Replacing $x$ and $y$ by $s$ and it in the above equation and taking the absolute values of the resulting equality one obtains the functional equation

$$
\left|f\left(\frac{s+i t}{2}\right)\right|=\left|\frac{f(s)+f(i t)}{2}\right| .
$$

The main purpose of this paper is to solve a new generalization of the above equation.

\section{INTRODUCTION}

We consider Jensen's functional equation

$$
f\left(\frac{x+y}{2}\right)=\frac{f(x)+f(y)}{2}
$$

(cf. [1, pp. 43-49, 139, 145, 286, 302; 2, pp. 142-146, 242-249, 255, 356-357; $3 ; 12]$ ) where $f$ is an unknown entire function of a complex variable $z$ and $x, y$ are complex variables.

One can prove the following theorem.

Theorem A. The only entire solution of (1) is given by $f(z)=A z+B$ where $A, B$ are arbitrary complex constants.

Proof. The proof is clear from operating $\partial^{2} / \partial y \partial x$ on both sides of (1) and setting $y=x$ in the resulting equality.

In what follows $f$ denotes an unknown entire function of a complex variable $\boldsymbol{Z}$.

Received by the editors April 30, 1993 and, in revised form, June 28, 1993.

1991 Mathematics Subject Classification. Primary 39B20.

Key words and phrases. Unknown entire function, Jensen's functional equation, Cosine functional equation, Robinson's functional equation, Hille's functional equation. 
In this paper we shall give a new generalization of (1). To this end we start with a few preliminary remarks. We consider the following two Cauchy equations (cf. [1, pp. 31-42; 2, pp. 11-24]) and the quadratic equation:

$$
f(x+y)=f(x)+f(y)
$$

and

$$
\begin{gathered}
f(x+y)=f(x) f(y), \\
f(x+y)+f(x-y)=2 f(x)+2 f(y),
\end{gathered}
$$

where $x, y$ are complex variables.

If we replace $x$ and $y$ by $s$ and it in (2) (3), (4), respectively, where $s, t$ are real variables, and we take the absolute values in the resulting equations, then we obtain the following three functional equations:

$$
\begin{aligned}
& |f(s+i t)|=|f(s)+f(i t)| \quad \text { (Robinson's functional equation; cf. [13]), } \\
& |f(s+i t)|=|f(s) f(i t)| \quad \text { (Hille's functional equation; cf. [10], [11]), }
\end{aligned}
$$

where $s, t$ are real variables, and

$$
|f(s+i t)+f(s-i t)|=2|f(s)+f(i t)| \quad(\text { cf. [8]). }
$$

In [6], it is proved that Robinson's functional equation is equivalent to the following Hille's functional equation (cf. $[10,11])$ :

$$
|f(s+i t)|^{2}=|f(s)|^{2}+|f(i t)|^{2},
$$

where $s, t$ are real variables.

For some recent generalizations of Hille's functional equation one may refer to $[5,7,14]$.

In a similar way, replacing $x$ and $y$ by $s$ and it in (1), where $s, t$ are real variables, and taking the absolute values of the resulting equality yields the functional equation

$$
\left|f\left(\frac{s+i t}{2}\right)\right|=\left|\frac{f(s)+f(i t)}{2}\right|,
$$

where $s, t$ are real variables.

In this paper we shall solve the functional equation (5). In fact, we shall prove the following theorem.

Theorem 1. The only entire solution of (5) is given by $f(z)=A z+B$ where $A, B$ are arbitrary complex constants.

In [9] the functional equation

$$
\left|f\left(\frac{s+i t}{2}\right)\right|^{2}=\left|\frac{f(s)+f(i t)}{2}\right|,
$$

where $s, t$ are real variables, was solved and the following theorem was proved. Theorem B. The only entire solutions of $(6)$ are given by $f(z) \equiv 0$ and

$$
f(z)=e^{i \theta} \cos (a z),
$$

where $\theta$ is an arbitrary real constant and $a$ is an arbitrary real or purely imaginary constant.

In the following we shall obtain a new generalization of (5) and (6), proving the following theorem: 
Theorem 2. The only entire solutions of the functional equations

$$
\left|f\left(\frac{s+i t}{2}\right)\right|^{m}=\left|\frac{f(s)+f(i t)}{2}\right|^{n},
$$

where $s, t$ are real variables and $m, n$ are arbitrarily fixed positive integers, are given as follows:

(i) If $m=n$, i.e., $m=1$ and $n=1$, the only entire solution of (7) is given by that in Theorem 1.

(ii) If $m=2 n$, i.e., $m=2$ and $n=1$, the only entire solutions of (7) are given by those in Theorem $\mathrm{B}$.

(iii) If $m \neq n$ and $m \neq 2 n$, the only entire solutions of (7) are complex constants with modulus 0 or 1 .

The purpose of this paper is to prove Theorem 2 by using Theorem 1 .

\section{Proofs of MAIN RESUlts}

To prove Theorems 1 and 2 we shall apply the following lemmas.

Lemma 1. If $f$ is an entire function of a complex variable $z$, then the conjugate function of $f$ denoted by $g$, i.e., $g(z)=\overline{f(\bar{z})}$, is also an entire function of $z$. Proof. Cf. [4, p. 28].

Lemma 2. Let $h, k$ be entire functions of a complex variable $z$, and let $p, q$ be arbitrarily fixed positive integers.

(i) If $h(z)^{p}=k(z)^{p}$ with $h(0)=k(0)=1$ for all complex $z$, then $h(z)=$ $k(z)$ for all complex $z$.

(ii) If $p \neq q$ and if $h(z)^{p}=h(z)^{q}$ with $h(0)=1$ for all complex $z$, then $h(z)=1$ for all complex $z$.

Proof. To prove (i) by the hypothesis that $h(z)^{p}=k(z)^{p}$ for all complex $z$ we obtain

$$
\prod_{j=0}^{p-1}\left(h(z)-w_{j} k(z)\right)=0
$$

for all complex $z$, where $w_{j}=\exp (2 j \pi i / p) \quad(j=0,1,2, \ldots, p-1)$.

Since the ring of all entire functions has no divisors of zero, then by (8) for some $j_{0}\left(0 \leq j_{0} \leq p-1\right)$, it follows that

$$
h(z)-w_{j_{0}} k(z)=0
$$

for all complex $z$.

Setting $z=0$ in (9) and using the hypothesis that $h(0)=k(0)=1$ yields

$$
w_{j_{0}}=1 \text {. }
$$

From (9), (10) we have $h(z)=k(z)$ for all complex $z$.

To prove (ii), by hypothesis we obtain

$$
h(z)^{p}=h(z)^{q}
$$

for all complex $z$.

Since, by hypothesis, $p \neq q$, we examine two cases. 
Case 1: Let $p>q$. By (11) we obtain

$$
\left(h(z)^{p-q}-1\right) h(z)^{q}=0
$$

for all complex $z$.

Since, by hypothesis, $h(0)=1$, we have

$$
h(z) \not \equiv 0 .
$$

Since the ring of all entire functions has no divisors of zero, by (13) we can divide both sides of (12) by $h(z)^{q}$. Hence we have $h(z)^{p-q}=1$, and therefore

$$
h(z)^{p-q}=1^{p-q}
$$

for all complex $z$.

By $(14), h(0)=1$, and by Lemma $2(\mathrm{i})$ we have $h(z)=1$ for all complex $z$.

Case 2: Let $p<q$. In this case the proof is similar to that of Case 1 .

Remark. The method of proving Theorems 1 and 2 is to introduce the conjugate function of $f$ (cf. Lemma 1) denoted by

$$
g(z)=\overline{f(\bar{z})}
$$

for all complex $z$.

In what follows let $g$ denote the conjugate function of $f$.

Proof of Theorem 1. We introduce the function $g$ which is the conjugate function of $f$ (cf. (15)). By Lemma $1, g$ is also an entire function of a complex variable $z$.

Our aim is to prove that $f^{\prime}(z)=$ const. Squaring both sides of (5) yields

$$
4\left|f\left(\frac{s+i t}{2}\right)\right|^{2}=|f(s)+f(i t)|^{2} \text {. }
$$

By the formula $|\gamma|^{2}=\gamma \bar{\gamma}$ for all complex $\gamma$ and by (16) we obtain

$$
4 f\left(\frac{s+i t}{2}\right) \overline{\left(\frac{s+i t}{2}\right)}=(f(s)+f(i t))(\overline{f(s)}+\overline{f(i t)}) \text {. }
$$

Replacing $z$ by $\bar{z}$ in (15) yields

$$
\overline{f(z)}=g(\bar{z})
$$

for all complex $z$.

By (18) we have

$$
\left\{\begin{array}{l}
\overline{f\left(\frac{s+i t}{2}\right)}=g\left(\frac{s-i t}{2}\right), \\
\overline{f(s)}=g(s), \\
\overline{f(i t)}=g(\overline{i t})=g(-i t) .
\end{array}\right.
$$

Substituting (19) into (17) yields

$$
4 f\left(\frac{s+i t}{2}\right) g\left(\frac{s-i t}{2}\right)=(f(s)+f(i t))(g(s)+g(-i t))
$$

for all real $s, t$. 
Here $f, g$ are entire functions. Hence, by (20) and by the Identity Theorem we have

$$
4 f\left(\frac{x+y}{2}\right) g\left(\frac{x-y}{2}\right)=(f(x)+f(y))(g(x)+g(-y))
$$

for all complex $x, y$.

Replacing $x, y$ by $x+y, x-y$ in (21), respectively, yields

$$
4 f(x) g(y)=(f(x+y)+f(x-y))(g(y+x)+g(y-x))
$$

for all complex $x, y$.

We may assume that

$$
f(z) \not \equiv 0 .
$$

Setting $x=0$ in (22) yields

$$
2 f(0) g(y)=(f(y)+f(-y)) g(y)
$$

for all complex $y$.

By (15) and (23) we obtain

$$
g(z) \not \equiv 0 .
$$

Since the ring of all entire functions has no divisors of zero, by (25) we can divide both sides of (24) by $g(y)$. Hence we have

$$
2 f(0)=f(y)+f(-y)
$$

for all complex $y$.

Differentiating both sides of (26) yields

$$
f^{\prime}(-y)=f^{\prime}(y)
$$

for all complex $y$.

Differentiating both sides of (22) with respect to $x$ and setting $x=0$ in the resulting equality yields

$$
2 f^{\prime}(0) g(y)=\left(f^{\prime}(y)+f^{\prime}(-y)\right) g(y)
$$

for all complex $y$.

Since the ring of all entire functions has no divisors of zero, by (25) we can divide both sides of $(28)$ by $g(y)$. Hence we have

$$
2 f^{\prime}(0)=f^{\prime}(y)+f^{\prime}(-y)
$$

for all complex $y$.

Substituting (27) into (29) yields

$$
f^{\prime}(y)=f^{\prime}(0)
$$

for all complex $y$.

By (30) we obtain

$$
f(y)=A y+B
$$

for all complex $y$, where $A=f^{\prime}(0)$ and $B$ is a complex constant.

Proof of Theorem 2. We examine three cases.

Case A. If $m=n$, i.e., $m=1, n=1$, the proof follows from Theorem 1 . 
Case B. If $m=2 n$, i.e., $m=2, n=1$, the proof follows from Theorem B.

Case C. Let $m \neq n$ and $m \neq 2 n$.

In this case we introduce the conjugate function $g$ (cf. (15)). By Lemma 1, $g$ is also an entire function of a complex variable $z$.

Our aim is to prove that $f$ is a complex constant with modulus 0 or 1 .

Squaring both sides of (7), using the formula $|\gamma|^{2}=\gamma \bar{\gamma}$ for all complex $\gamma$, and applying a similar method to that in the proof of Theorem 1 yields

$$
4^{n}(f(x) g(y))^{m}=((f(x+y)+f(x-y))(g(y+x)+g(y-x)))^{n}
$$

for all complex $x, y$.

Setting $s=t=0$ in (7) and using the hypothesis $m \neq n$ yields $f(0)=0$ or $|f(0)|=1$.

We discuss the two subcases of Case $C$.

Case $C_{1}$ : Let $f(0)=0$. Setting $z=0$ in (15) and using $f(0)=0$ yields

$$
g(0)=0 \text {. }
$$

Setting $y=x$ in (31) and using $f(0)=0$ and (32) yields

$$
4^{n}(f(x) g(x))^{m}=(f(2 x) g(2 x))^{n}
$$

for all complex $x$.

$f$ and $g$ are entire functions. Therefore, if we set

$$
l(x)=f(x) g(x)
$$

for all complex $x$, then $l$ is also an entire function.

Our aim is to prove that $l(x) \equiv 0$. Assume that this is not the case. Then $l(x) \not \equiv 0$. Furthermore, $l$ is an entire function. Hence, $l$ has the power series expansion of the form

$$
l(x)=\sum_{m=r}^{+\infty} l_{m} x^{m}
$$

for all complex $x$, where $l_{r} \neq 0$ and $r$ is a positive integer by $l(0)=0$ which follows from $f(0)=0$ and (34).

By (33) and (34) we obtain

$$
4^{n} l(x)^{m}=l(2 x)^{n}
$$

for all complex $x$.

By hypothesis we have $m \neq n$.

We consider two subcases of $C_{1}$.

Case $C_{11}$ : Let $m<n$.

Substituting (35) into (36) and equating the coefficients of $x^{m r}$ yields $4^{n} l_{r}^{m}=$ 0 , and so $l_{r}=0$, which contradicts the fact that

$$
l_{r} \neq 0 \text {. }
$$

Case $C_{12}$ : Let $m>n$. Similarily, as above, we arrive at the contradiction. Hence

$$
l(x) \equiv 0 \text {. }
$$

From (15), (34), and (37) we obtain $f(z)=0$ for all complex $z$. 
Case $C_{2}$ : Let $|f(0)|=1$. In this case we normalize $f$. We define $\theta$ to be

$$
\theta \stackrel{\text { def }}{=} \arg (f(0)) \text {. }
$$

If we set

$$
F(z) \stackrel{\text { def }}{=} e^{-i \theta} f(z)
$$

for all complex $z$, it follows from (7) and (39) that $F$ satisfies the functional equation

$$
\left|F\left(\frac{s+i t}{2}\right)\right|^{m}=\left|\frac{F(s)+F(i t)}{2}\right|^{n}
$$

for all real $s, t$ and, by the hypothesis $|f(0)|=1$ and (38) and (39), that

$$
F(0)=1 \text {. }
$$

Now we introduce the conjugate function of $F$ denoted by $G$, i.e.,

$$
G(z)=\overline{F(\bar{z})}
$$

for all complex $z$.

By a similar method of getting (22) from (15) we get from (41)

$$
4^{n}(F(x) G(y))^{m}=\left((F(x+y)+F(x-y))(G(y+x)+G(y-x))^{n}\right.
$$

for all complex $x, y$.

Setting $z=0$ in (43) and using (42) yields

$$
G(0)=1 \text {. }
$$

Our aim is to prove that $F(z) \equiv 1$.

Setting $x=0$ in (44) and using (42) gives

$$
2^{n} G(y)^{m}=(F(y)+F(-y))^{n} G(y)^{n}
$$

for all complex $y$.

We discuss two subcases of $C_{2}$.

Case $C_{21}:$ Let $m<n$. By (45) we have

$$
G(y) \not \equiv 0 .
$$

Since the ring of all entire functions has no divisors of zero, by (47) we can divide both sides of (46) by $G(y)^{m}$. Hence we have

$$
2^{n}=(F(y)+F(-y))^{n} G(y)^{n-m}
$$

for all complex $y$.

Replacing $y$ by $-y$ in (48) yields

$$
2^{n}=(F(-y)+F(y))^{n} G(-y)^{n-m}
$$

for all complex $y$.

Dividing (48) by the above equality yields

$$
G(-y)^{n-m}=G(y)^{n-m}
$$

for all complex $y$. 
Since $G(-y), G(y)$ are entire functions of $y$ with $G(-0)=G(0)=1$ which follows from (45), by (49), the fact that $n-m$ is a positive integer, and by Lemma 2(i), we obtain

$$
G(-y)=G(y)
$$

for all complex $y$.

By (43) and (50) we obtain

$$
F(-y)=F(y)
$$

for all complex $y$.

By (48) and (51) we have

$$
F(y)^{n} G(y)^{n-m}=1
$$

for all complex $y$.

By the symmetry property of $F$ and $G$ we can interchange $F$ and $G$ in (52). So we obtain

$$
G(y)^{n} F(y)^{n-m}=1
$$

for all complex $y$.

Dividing (52) by (53) side by side yields

$$
F(y)^{m}=G(y)^{m}
$$

for all complex $y$.

Since $F(y), G(y)$ are entire functions of $y$ with $F(0)=G(0)=1$ which follows from (42), (45), by (54), and by Lemma 2(i), we obtain

$$
F(y)=G(y)
$$

for all complex $y$.

By (52) and (54) we have

$$
F(y)^{2 n-m}=1^{2 n-m}
$$

for all complex $y$.

Since $F(y)$ is an entire function of $y$ with $F(0)=1$, by (56), the fact that $2 n-m$ is a positive integer, and by Lemma 2(i), we have

$$
F(y)=1
$$

for all complex $y$, i.e., $F(z) \equiv 1$.

Case $C_{22}$ : Let $m>n$ with $m \neq 2 n$. Dividing both sides of (46) by $G(y)^{n}$ and getting $F(-y)=F(y)$ for all complex $y$ by using a similar method to that in Case $C_{21}$ yields

$$
G(y)^{m-n}=F(y)^{n}
$$

for all complex $y$.

By the symmetry property of $F$ and $G$ we can interchange $F$ and $G$. Hence, we obtain $F(y)^{m-n}=G(y)^{n}$, so

$$
G(y)^{n}=F(y)^{m-n}
$$

for all complex $y$.

Multiplying (57) and (58) side by side yields $F(y)^{m}=G(y)^{m}$ for all complex $y$. 
Consequently, we obtain

$$
F(y)=G(y)
$$

for all complex $y$ (cf. (54), (55)).

By (57) and (59) we obtain

$$
F(y)^{m-n}=F(y)^{n}
$$

for all complex $y$.

Since, by hypothesis, $m \neq 2 n$, the two positive integers $m-n, n$ are different. Furthermore, $F(0)=1$. Hence, by (60) and Lemma 2(ii) we have $F(y) \equiv 1$, for all complex $y$, i.e., $F(z) \equiv 1$.

Thus we have established

$$
F(z) \equiv 1 \text {. }
$$

By (40) and (61) we obtain $f(z) \equiv e^{i \theta}$, where $\theta$ is a real constant.

Consequently, $f$ is a complex constant with modulus 0 or 1 .

\section{REFERENCES}

1. J. Aczél, Lectures on functional equations and their applications, Academic Press, New York and London, 1966.

2. J. Aczél and J. Dhombres, Functional equations in several variables, Cambridge Univ. Press, Cambridge, New York, New Rochelle, Melbourne, and Sydney, 1989.

3. J. Aczél and E. Vincze, Über eine gemeinsame Verallgemeinerung zweier Funktionalgleichungen von Jensen, Publ. Math. Debrecen 10 (1963), 326-344.

4. L. V. Ahlfors, Complex analysis, 2nd ed., McGraw-Hill, New York, 1966.

5. Boo Rim Choe, A functional equation of Pexider type, Funkcial. Ekvac. 35 (1992), 255-259.

6. H. Haruki, On the equivalence of Hille's and Robinson's functional equations, Ann. Polon. Math. 28 (1973), 261-264.

7. _ On a functional equation of Pexider type, Aequationes Math. 36 (1988), 1-19.

8. A new quadratic equation, Constantin Caratheódory: An International Tribute (Th. M. Rassias, ed.), World Scientific, Singapore, New Jersey, and London, 1991, pp. 476-488.

9. A new cosine functional equation, The Mathematical Heritage of C. F. Gauss (G. M. Rassias, ed.), World Scientific, Singapore, New Jersey, and London, 1991, pp. 334-341.

10. E. Hille, $A$ Pythagorean functional equation, Ann. of Math. (2) 24 (1923), 175-180.

11. __ A class of functional equations, Ann. of Math. (2) 29 (1928), 215-222.

12. C. $\mathrm{T}$. $\mathrm{Ng}$, The Jensen equation on groups, Aequationes Math. 37 (1989).

13. R. M. Robinson, A curious trigonometric identity, Amer. Math. Monthly 64 (1957), 83-85.

14. Problems and solutions section, Amer. Math. Monthly 99 (1992), 875.

Department of Pure Mathematics, Faculty of Mathematics, University of Waterloo, Waterloo, Ontario, Canada N2L 3G1

Department of Mathematics, University of La Verne, P.O. Box 51105, Kifissia, Athens, GREECE 14510 